\title{
Tanaka/Johnston - Um método simplificado e efetivo para a predição de tamanho de dentes permanentes não erupcionados
}

\section{Tanaka/Johnston - An efective and simplified method for the prediction of the size of not erupted teeth}

\author{
Telmo Bandeira Berthold* \\ Luciane Q. Closs** \\ Paulo Pan Nys** \\ Joåo Antônio Caminha***
}

\section{Resumo:}

Em um estudo feito em 52 pares de modelos de gesso de pacientes leucodermas, naturais de Porto Alegre, foi medido o diâmetro M-D de premolares e caninos permanentes com a finalidade de testar o método de Tanaka/Johnston ${ }^{14}$. Neste estudo, nåo foram encontradas diferenças estatisticamente significantes entre os valores reais es previstos por este método para as arcadas superior $\theta$ inferior do sexo masculino e para a arcada superior do sexo feminino. $\mathrm{Na}$ arcada inferior do sexo feminino houve uma pequena diferença estatisticamente significante ( $p=0.01)$.

\section{Summary:}

In a study made in $\mathbf{5 2}$ pairs of cast models of white patients native to Porto Alegre, measures of the M-D diameters of permanent cuspids and bicuspids were taken, with the objective of testing Tanaka/Johnston's method ${ }^{14}$. In this study, no significant statiscal diference was found between the real values and the ones predicted by this method for both upper and lower arch for males and of upper arch for females. In the lower arch females, a small significant statistical diference $(p=0,01)$ was found.

\section{Introdução:}
$\Delta$ predição do diâmetro de cani- nos e pre-molares na fase de dentiçăo mista tem-se tornado cada vez mais necessária para um correto diagnóstico. Na literatura, encon- tram-se diversos métodos, os quais baseiam-se em radiografias (periapi- cais, telerradiografia em $45^{\circ}$ ), da- dos estatísticos (Moyers ${ }^{10}$, Tanaka/ Johnston ${ }^{14}$ ) e outros (Huckaba7, Nance ${ }^{11}$ ). Após uma detalhada análise de alguns destes métodos, resolveu-se fazer uma pesquisa em

pacientes leucodermas, naturais de Porto Alegre, para testar o Método de Tanaka/Johnston ${ }^{14}$. O método demonstrou uma boa confiabilidade e chamou a atenção pela sua simplicidade, rápida utilização e baixo custo.

\section{Descrição do método} Tanaka/Johnston:

5te método tem como ponto de
partida o somatório dos diâma-
tros M-D dos incisivos permanentes inferiores. Para fazer-se a predição do espaço necessário para a correta disposiçăo dos dentes permanentes (caninos e pré-molares), procede-se da seguinte maneira: divide-se por dois o somatório dos diâmetros dos quatro incisivos inferiores e soma-se a um valor pré- determinado que corresponde a $10,5 \mathrm{~mm}$ para a mandíbu-

* Professor de Ortodontia - PUC/RS e UFRGS.

* Cirurgióes-dentistas, ex-intermos Ortodontia/UFRGS.

** Diretor e Professor da Faculdade de Odonto logia/UFRGS.

R. Fac. Odontol.

Porto Alegre

v. 34

N. 1

p.28-31

SETEMBRO

1993 
la e $11,0 \mathrm{~mm}$ para a maxila. Esta soma multiplicada por dois, equivale ao valor previsto para o diâmetro dos dentes mencionados acima para as duas hemi-arcadas inferiores e superiores, respectivamente.

\section{Exemplo:}

Um paciente que apresente o somatório $M-D$ dos dentes $31,32,41$, 42 igual a $24,0 \mathrm{~mm}$ procede-se da seguinte maneira: divide-se este valor por dois ficando em $12,0 \mathrm{~mm}$ e somase a 10,5 para a arcada inferior. Este valor, $22,5 \mathrm{~mm}$ é multiplicado por dois e o valor encontrado, $45,0 \mathrm{~mm}$, será a quantidade de espaço previsto para o correto pcsicionamento dos caninos e pré-molares inferiores em ambos os lados. Para a arcada superior procede-se da mesma maneira, mas ao invés de somar-se 10,5 , so ma-se 11,0 . Soma-se entåo $12,0 \mathrm{~mm}$ mais 11,0 e multiplica-se por 2 . Este valor, $46 \mathrm{~mm}$, será o previsto para o correto posicionamento de caninos e pré-molares superiores no lado esquerdo e direito. Desta forma, no cálculo da discrepância de modelo $D M=E P-E R$, o espaço requerido inferior seria: $45 \mathrm{~mm}$ (obtido atraves do metodo de Tanaka/Johnston) + $22 \mathrm{~mm}$ que é a soma dos incisivos inferiores. Para a arcada superior o espaço requerido seria a soma de $46 \mathrm{~mm}$ (obtidos através do método de Tanaka/Johnston) mais a soma $M-D$ dos incisivos superiores.

INFERIOR: $32,31,41,42=24 \mathrm{~mm}$

$$
\begin{gathered}
\left(\sum \frac{2,11,2}{2}+10,5\right) \times 2=\begin{array}{c}
\text { VALOR } \\
\text { PREVISTO }
\end{array} \\
\left(\frac{24}{2}+10,5\right) \times 2=45 \mathrm{~mm}
\end{gathered}
$$

* Previsão para o diåmetro M-D dos dentes 33, 34, 35, 43, 44, $45=45$ $\mathrm{mm}$

\section{SUPERIOR:}

$$
\begin{gathered}
\left(\sum \frac{2,11,2}{2}+11,0\right) \times 2=\begin{array}{c}
\text { VALOR } \\
\text { PREVISTO }
\end{array} \\
\left(\frac{24}{2}+11,0\right) \times 2=46 \mathrm{~mm}
\end{gathered}
$$

- Previsåa para o diâmetro M-D dos dentes $13,14,15,23,24,25=46$ $\mathrm{mm}$

\section{DIÂMETRO M - D \\ Masculino}

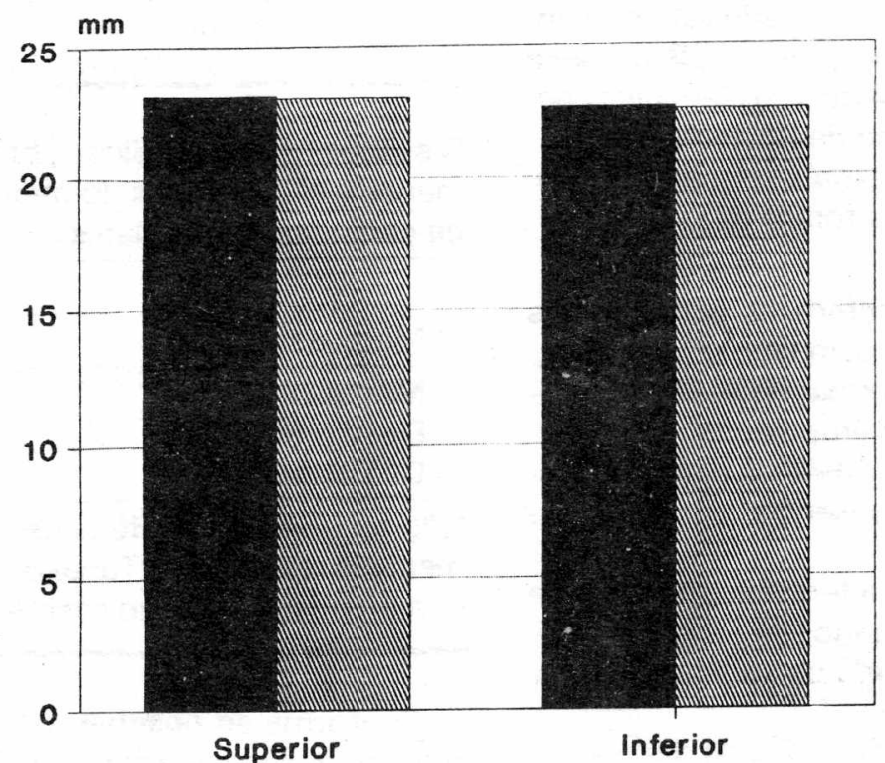

Média

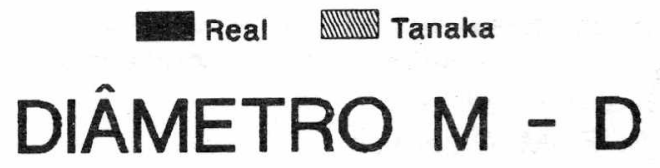

Feminino

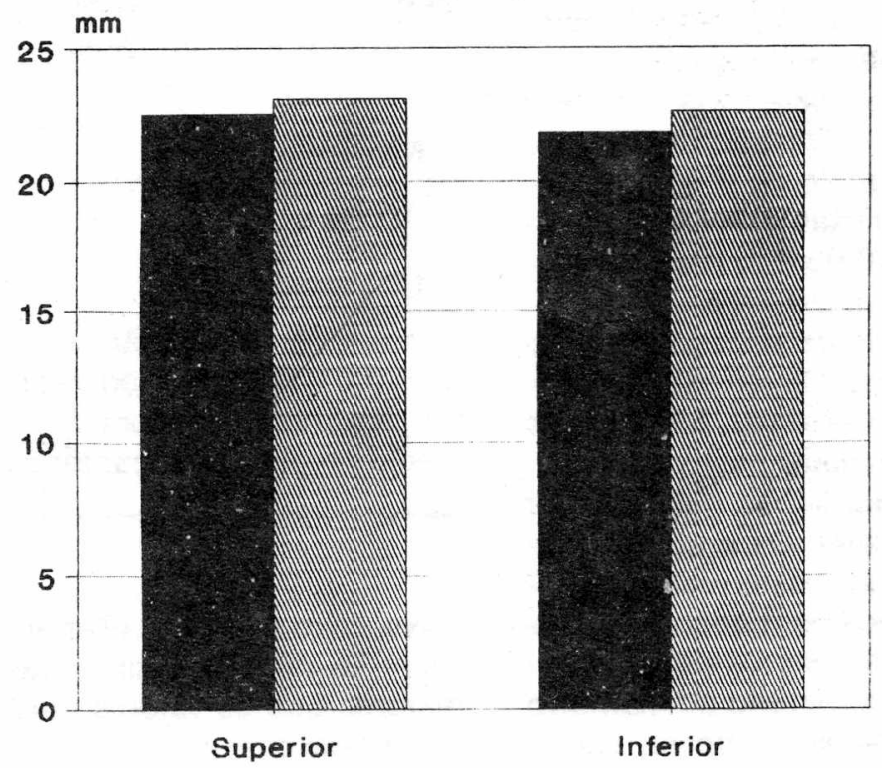

Média

Real Whilly Tanaka 


\section{Material e método:}

$\mathbf{F}$ oram selecionados 52 pares de modelo de gesso de pacientes leucodermas, com idade variando entre 11 e 17 anos, sendo 28 do sexo masculino e 24 do sexo feminino, naturais da cidade de Porto Alegre.

Os critérios para a seleção dos modelos dentários foram os seguintes:

- Incisivos inferiores permanentes totalmente erupcionados;

- Caninos permanentes e pré-molares totalmente erupcionados;

- Não haver ausência dentária congênita, nem ausência por cárie ou traumatismo;

- Não haver alterações dentárias de forma ou tamanho (de ordem congênita ou por perda de substância: atrição ou cárie);

- Presença de todos os dentes envolvidos na pesquisa.

As medidas foram tomadas diretamente nos modelos de gesso com o auxílio de um compasso de ponta seca, de marca DENTAURUM. O compasso foi posicionado o mais perpendicular possível em relação ao longo eixo do dente e no maior diâmetro da coroa. Primeiramente, dois examinadores registraram independentemente duas medidas para cada caso. Foi efetuada uma calibração dos examinadores no sentido de evitar diferenças intra e inter examinadores e foi estabelecido em $0,05 \mathrm{~mm}$ o valor máximo de diferença. Discrepâncias maiores que este limite necessitaram de novas medidas. Os arredondamentos, quando necessários, foram feitos sempre para a média mais próxima.

Foram separadas as medidas por sexo e por arcada. Os grupos medidos foram: incisivos permanentes inferiores, caninos permanentes e prémolares de cada hemi-arco.

A partir do somatório do diametro $M-D$ dos incisivos inferiores, calculou-se a previsão do espaço para caninos e prémolares segundo o método de Tanaka/Johnston.

\section{Resultados:}

A Tabela 1 mostra os dados obtidos para o sexo masculino, onde verifica-se a média, o desvio pa- drão e o coeficiente de variação para as medidas reais e as estimadas pelo método de Tanaka/Johnston ${ }^{14}$.

\section{TABELA 1}

Distribuição das medidas obtidas em 28 pacientes do sexo masculino, diretamente e as predições do método de Tanaka/Johnston, para o diâmetro M-D de caninos e pré-molares.

\begin{tabular}{lrrrr}
\hline & \multicolumn{2}{c}{ Real } & \multicolumn{2}{c}{ Tanaka/Johnston } \\
\cline { 2 - 5 } Medidas & Sup. & \multicolumn{1}{c}{ Inf. } & \multicolumn{1}{c}{ Sup. } & \multicolumn{1}{c}{ Inf. } \\
\hline Média $(x)$ & 23,13 & 22,66 & 23,05 & 22,55 \\
Desvio Padrão(s) & 0,92 & 0,83 & 0,61 & 0,61 \\
C. Variação \% & 3,98 & 3,66 & 2,65 & 2,71 \\
\hline
\end{tabular}

Não foi observada diferença estatisticamente significante $(p=0,01)$ entre as medidas reais e as de Tanaka/ Johnston, tanto para a arcada superior como para a arcada inferior no sexo masculino.

Conforme se observa, os valores médios reais são muito próximos aos dos valores médios previstos pelo método de Tanaka/Johnston. Nota-se ainda, que o valor real é pouca coisa maior $(0,11 \mathrm{~mm})$ que o previsto, nos dentes inferiores.

Verifica-se também, que o coeficiente de variação apresenta baixas percentagens, sendo que o maior foi de $3,98 \%$ no caso da medida direta nos dentes superiores.

\section{TABELA 2}

Distribuição das medidas obtidas em 24 pacientes do sexo feminino, diretamente e as estimativas do método de Tanaka/Johnston, para o diâmetro M-D de caninos e pré-molares.

\begin{tabular}{lrrrr}
\hline & \multicolumn{2}{c}{ Real } & \multicolumn{2}{c}{ Tanaka/Johnston } \\
\cline { 2 - 5 } Medidas & Sup. & \multicolumn{1}{c}{ Inf. } & \multicolumn{1}{c}{ Sup. } & \multicolumn{1}{c}{ Inf. } \\
\hline Média $(\overline{\mathrm{x}}$ ) & 22,50 & 21,83 & 23,10 & 22,60 \\
Desvio Padrão(s) & 1,73 & 1,12 & 2,63 & 0,69 \\
C. Variação \% & 8,97 & 5,13 & 11,39 & 3,05 \\
\hline
\end{tabular}

O sexo feminino não apresentou diferença estatisticamente significante $(p=0,01)$ entre as medidas reais e as de Tanaka/Johnston para a arcada superior. Na arcada inferior a diferença é estatisticamente significante $(p=0,01)$. A medida real é significantemente inferior a de Tanaka/Johnston.

No sexo feminino (Tabela 2), observa-se que as estimativas foram maiores que os valores medidos diretamente para as arcadas superior $e$ inferior. A variaçăo das medidas no sexo feminino foram maiores que as encontradas no sexo masculino, sendo a arcada superior no método de Tanaka/Johnston, a medida que apresentou maior variabilidade $(11,39 \%)$, seguida da medida direta, também na arcada superior, que foi de $8,97 \%$. 


\section{Conclusões:}

ão existem diferenças estatisticamente significativas $(p=$
$0,01)$ entre os valores reais e os previstos pelo método de Tanaka/ Johnston, com exceção às medições da arcada inferior no sexo feminino; contudo, como esta medida prevista é levemente maior que o valor real, não acarreta em nenhum prejulzo clínico significativo. A facilidade de uso do Método de Tanaka/Johnston e a sua confiabilidade clínica, são fatores que o diferenciam dos demais métodos.

\section{Bibliografia:}

1. BALLARD, M.L. \& WYLIE, W.L. - Mixed dentition case analysis. Estimating size of unerupted permanent teeth Amer. J. Orthdont., 33:754-9, 1947.

2. BERTHOLD et ali. - Avaliação dos métodos de predição do diâmetro de caninos e prémolares não erupcionados de Moyers e o de Tanaka/ Johnston. Ortodontia Paranaense, Curitiba - v. 13. n.1, p. 1-6, 1992.

3. BISCHARA, S.E. - Compensatory development interactions in the size of permanent teeth in contemporary population. Angle Orthodontist, 59-2:107-111, 1989.

4. BLACK, G.V. - Descriptive anatomy of human teeth. Ed. 4. Philadelphia, 1902. S.S. White Dental Mifg. Co.

5. BOLTON, W.A. - The clinical aplication of a tooth size analysis. Amer. J. Orthodont., 48:504-29, 1962.
6. GARDNER, R.B. - A comparision of four methods of predicting arch lenght. Amer. J. Orthodont., 75:387-98. 1979.

7. HUCKABA, G.W. - Arch size analysis and tooth size prediction. Dent. Clin. North. Am. 11:431-40, 1964.

8. MOORREES, C.F.A. \& REED R.B. Correlation among crown diameters of human teeth. Arch Oral Biol. 9:68597, 1964.

9. MOORREES, C.F.A. et ali. Mesiodistal crown diameters of deciduous and permanent teeth in individuals. J. Dent. Res. 36-39, 1957.

10. MOYERS, R.E. - Ortodontia 3: ed. Rio de Janeiro, Guanabara. Koogan. 308-40, 1979.

11. NANCE; M.L. - Limitations of orthodontic treatment I Diagnosis and treatment in the mixed dentition. Am. J. Orthodont. • Oral Surg. 33: 177-223, 1947.
12. STAleY, R.N. et alli. - Prediction of lower canine and premolar widths in the mixed dentition. Amer. J. Orthodont., 76:300-9, 1979.

13. STAHLE, H. - Determination of mesiodistal crown width of unerupted cuspid and bicuspid. Helv. Odont. Acta. 3:14-7, 1959.

14. TANAKA, M.M. \& JOHNSTON, L.E. - The prediction of the size of unerupted canines and pre-molars in a contemporary orthodontic population. J. Amer. Dent. Ass., 88:798801, 1974.

15. WATSON, D.H. - A rapid and acurate assesment of the mesiodistal dimensions of any unerupted permanent teeth. Int. J. Orthod., 10:91-2, 1972.

16. ZILBERMAN, V. et ali . - Estimating of mesiodistal widht of permanent canines and premolars in early mixed dentition. J. Dent. Res., 66:911-5, 1977. 